\title{
Design of a vacuum based test rig for measuring micro adhesion and friction force
}

\author{
M. A. Yaqoob ${ }^{1,2}$, M. B. de Rooij ${ }^{2}$ \& D. J. Schipper ${ }^{2}$ \\ ${ }^{I}$ Materials Innovation Institute, Delft, The Netherlands. \\ ${ }^{2}$ Department of Engineering Technology, University of Twente, \\ Enschede, The Netherlands
}

\begin{abstract}
Mechanisms operating in low pressure (vacuum), nitrogen or other special environments are found in many applications. Examples are medical instrumentation, electron microscopes, lithography systems, as well as aviation and space applications. The positioning accuracy and drifts in these mechanisms are strongly influenced by the frictional behaviour of the mating materials. The cause for both drift and positioning accuracy are stick-to-slip and slip-to-stick transitions at asperity level, resulting in a displacement at macrolevel. This paper discusses the experimental setup designed and manufactured to validate a model which relates friction and positioning accuracy for a particular pair of material at asperity level. A simplified form of a single asperity contact is a ball in contact with a flat surface. The experimental setup has been designed to validate the friction and adhesion models. Snap-in, pull-off experiments and friction force measurements can be performed with the resolution of $5 \mu \mathrm{N}$. The maximum normal load that can be applied with this system is $100 \mathrm{mN}$. The setup is capable of working at $10^{-6}$ mbar vacuum level. A 2 Degree of Freedom (DOF) elastic hinge mechanism is the heart of the setup and measures the normal and tangential load with the help of capacitive displacement sensors. In the setup, high precision positioning stages have been used which are capable of moving $20 \mathrm{~mm}$ in XYZ with an accuracy of $20 \mathrm{~nm}$. The setup is also able to perform friction measurements with the same accuracy as mentioned above. Design and performance of the setup will be discussed and the results are compared with the theory.

Keywords: adhesion force, friction force, force-displacement curves, positioning accuracy, high vacuum, mechanical vibrations.
\end{abstract}




\section{Introduction}

Many practical macroscopic engineering applications involving tribology have been successfully addressed in recent years, but relatively little is understood. Fundamental mechanisms of adhesion, friction and energy dissipation between sliding surface are one of the important areas of research for tribologists and physicists in the modern era. With the advent of Atomic Force Microscope (AFM), Friction Force Microscope (FFM) and Surface Force Apparatus (SFA) a lot has been contributed to the understanding of friction at nanoscale, see Israelachivili [1]. However, the AFM is typically used for adhesion and friction measurements typically with nanometer sized tips and normal load of few hundreds of $\mathrm{nN}$. On the other hand SFA is used to measure the adhesion forces with smooth surfaces with typical tip radius of $1 \mathrm{~cm}$, see Israelachivili [1]. The use of nanometer-sharp AFM tips in friction measurements hinders the quantitative comparison between the experimental data and the contact model calculations for elastically deforming interfaces (Grobelny et al. [2]).

Knowledge on frictional behaviour of positioning mechanisms and its relation to positioning accuracy is scarce, especially when they are operating in special environments as vacuum and (dry) nitrogen. Friction, as related to positioning accuracy, is often studied for control purposes. To develop more accurate control algorithms friction is taken as a part of the dynamical system, thus, it makes the control algorithms complicated and non-linear in nature. An extensive number of models have been developed for this purpose as explained by AmstrongHélouvry [3] and Capone et al. [4]. Such models are typically aimed at modelling effects like a velocity dependency of dynamic friction.

Values of the coefficient of friction have been reported for countless couples of materials under different conditions, from liquid ambient to ultra-high vacuum conditions. Nevertheless, to our knowledge there is no physical model which can relate the coefficient of friction to the fundamental concept of surface forces and positioning accuracy. In case of micro and nano scale experiments the fundamental laws of friction are not obeyed and the system is dominated by adhesion-influenced contact mechanics, see Feldman et al. [5].

Adhesion force is present between two surfaces when brought closer or made contact with each other. This force is generally caused by the superposition (expressed in eqn. (1) Xiao and Qian [6]) of different kind of surface forces like van der Waals forces, electrostatic forces, capillary forces and other interacting surface forces Israelachvili [1].

$$
F_{a}=F_{v d w}+F_{c a p}+F_{e l}+\ldots
$$

where, $F_{a}(N)$ is adhesion force, $F_{v d w}(N)$ is van der Waals force, $F_{c a p}(N)$ is capillary force and $F_{e l}(N)$ is electrostatic force. The adhesion force strongly depends on many physical factors like surface energy, surface roughness, geometry and size, separation distance, applied normal load, environmental conditions like pressure and temperature, duration of contact, hydrophilic or hydrophobic nature of the contacting surfaces, see Bhushan [7]. 
Different theories and models have been developed to understand the relationship between friction and adhesion at micro and nano scale, see Johnson and Greenwood [8]. Johnson, Kendall and Roberts (JKR), Derjaguin, Muller and Topolov (DMT), Tabor and Maugis-Dugdale (MD) presented the contact mechanics models for elastic deformation in the contact incorporating adhesion force. Furthermore, the work of adhesion in the contact can be calculated by measuring the adhesion force or pull-off force and using the applicable contact model for the system, see Johnson and Greenwood [8].

In this paper, the experimental setup designed and manufactured for performing adhesion and friction measurements at micro level in different environments like ambient air, dry nitrogen atmosphere and high vacuum is discussed. Measurements which have been performed to characterize the performance of the test rig, both in ambient air and vacuum, are also discussed and the results are compared with the theory.

\section{Design}

A complete new vacuum based test rig was designed, manufactured, assembled and tested. The main aim of this setup is to perform adhesion and friction measurements at micro scale in ambient $\left(20^{\circ} \mathrm{C}, 1\right.$ bar and $50 \%$ Relative Humidity) as well as in special environments like high vacuum $\left(20^{\circ} \mathrm{C}\right.$ and $\left.10^{-6} \mathrm{mbar}\right)$ and dry nitrogen. This Vacuum Adhesion and Friction Tester (VAFT) comprises of three positioning stages and two capacitive sensors along with a force measuring mechanism as shown in fig. 1. The setup has a ball on flat configuration and represents a single asperity contact. The ball is mounted on the indenter and the indenter along with the force measuring mechanism is mounted on one of the positioning stages which can move in $\mathrm{Z}$ direction as shown in fig. 1. This positioning stage is used to make contact with the flat surface and to apply normal load. The flat surface is placed on an XY stage. The X positioning stage is used to apply a tangential load for friction measurements. The accuracy of both $\mathrm{X}$ and $\mathrm{Z}$ stage is $20 \mathrm{~nm}$ with a stroke of $20 \mathrm{~mm}$. The Y stage is used to perform multiple parallel measurements on the flat surface and has a stroke of $20 \mathrm{~mm}$ as well.

The measuring range of the capacitive sensors is $50 \mu \mathrm{m}$ with accuracy better than $1 \mathrm{~nm}$. The capacitive sensors are mounted on the force measuring mechanism. The stiffness of the force measuring mechanism is calibrated, and then by measuring the deflection of this mechanism with the help of capacitive sensors, the force can be calculated.

\subsection{Force measuring mechanism}

The heart of the VAFT is the force measuring mechanism. The mechanism is designed to have two DOFs for measuring normal and friction force. The conceptual design of this mechanism has been explained by Awtar [9]. It was required to have a mechanism which can measure the two perpendicular forces independently as much as possible. The mechanism consists of 8 compound parallelogram frictionless hole-hinge flexure mechanisms. 
264 High Performance Structure and Materials VI

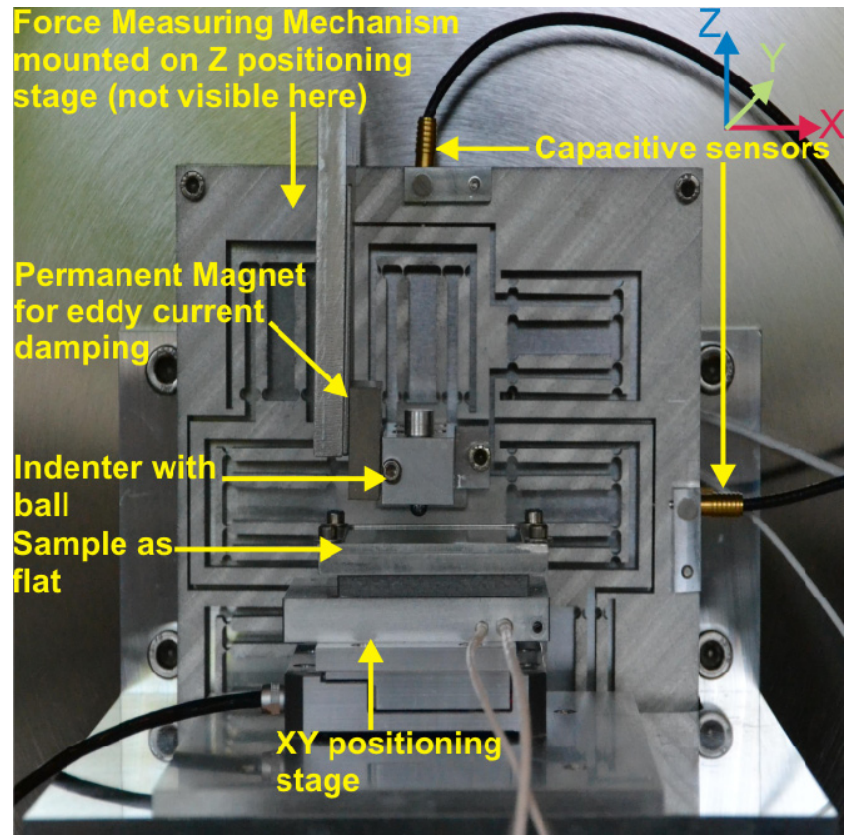

(a)

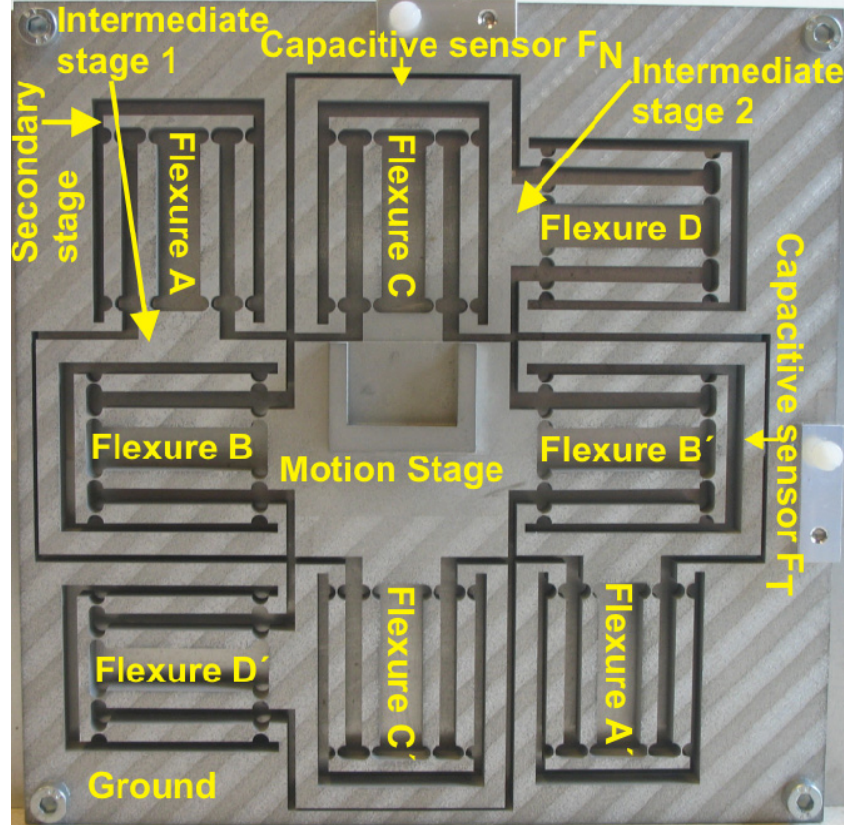

(b)

Figure 1: (a) Internal view of VAFT showing all the components. (b) Illustration of force measuring mechanism. 
In the mechanism, there are four rigid stages: ground, motion stage, and two intermediate stages as shown in fig. 1(b). The intermediate stages are necessary to decouple and isolate the motion of the two axes. The four compliant units are called Flexure A, B, C and D and their respective mirrored compliant units are Flexure $\mathrm{A}^{\prime}, \mathrm{B}^{\prime}, \mathrm{C}^{\prime}$ and $\mathrm{D}^{\prime}$. When the normal force is applied the Flexure B, B' and $\mathrm{D}, \mathrm{D}^{\prime}$ would bend to give the desired displacement and Flexure $\mathrm{A}, \mathrm{A}^{\prime}$ and $\mathrm{C}$, $\mathrm{C}^{\prime}$ are in tensile/compressive load. Similarly, when the lateral force is applied flexure $\mathrm{A}, \mathrm{A}^{\prime}$ and $\mathrm{C}, \mathrm{C}^{\prime}$ deflects to give the desired motion and Flexure B, B' and $\mathrm{D}, \mathrm{D}^{\prime}$ are in tensile/compressive load. Any parasitic errors due to bending of compound flexures are compensated by the secondary motion stage. Furthermore, this force measuring mechanism is relatively insensitive to thermal disturbances and manufacturing errors due to its symmetry.

The force measuring mechanism has been calibrated and the stiffness of the mechanism has been calculated. In fig. 2 the deflection of the hinges measured by the capacitive sensor on the application of normal load is shown. The calibrated stiffness of the force measuring mechanism calculated by the slope of the force-displacement curve of fig. 2 is $3.75 \mathrm{mN} / \mu \mathrm{m}$.

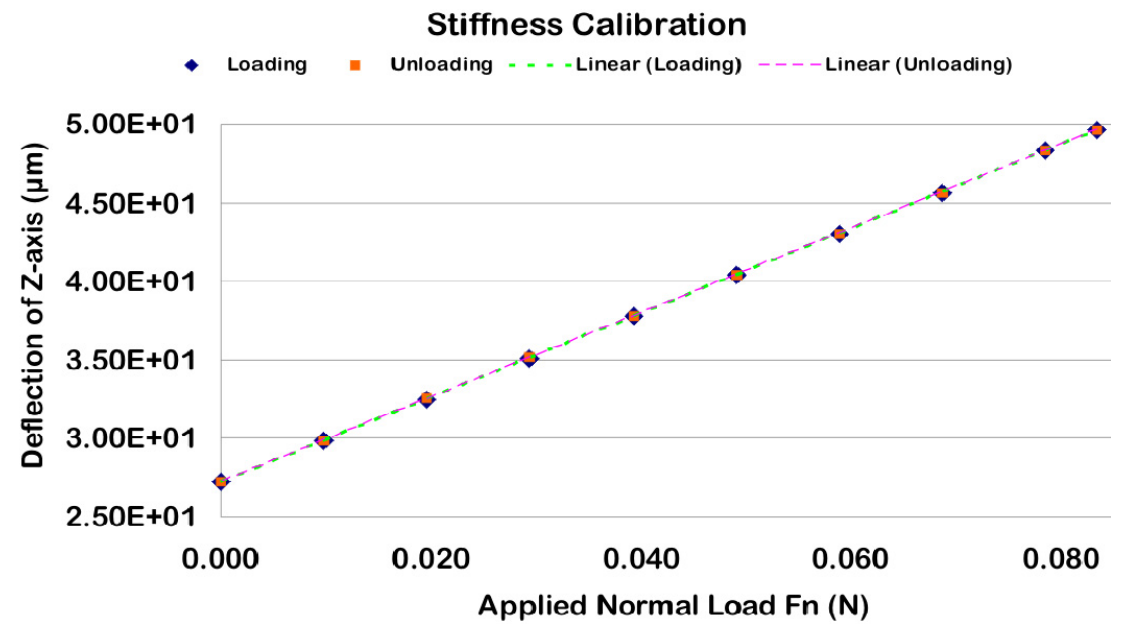

Figure 2: Force-displacement curve for calibrating the stiffness of the force measuring mechanism.

\subsection{Vibration isolation}

The complete system is mounted inside a vacuum chamber as shown in fig. 3 . Mechanical disturbances are the major potential source of instability and inaccuracy in the system. These disturbances are mainly categorized as ground vibrations, vibrations from the vacuum pump and vibrations induced due to dynamic effects of the moving $Z$ positioning stage. The ground vibrations are caused by the traffic around the lab and the structural vibrations of the building. These vibrations are reduced by placing air pads under the platform carrying the vacuum chamber as shown in fig. 3(c). The vibrations from the vacuum pumps 
are reduced by placing the backing pump on a separate platform than the vacuum chamber and the turbo pump. The turbo pump is connected to the chamber via an anti-vibration bellows which reduces the vibration amplitude by a factor of 10 as explained in [10]. The vibrations induced by the $Z$ positioning stage are diminished by introducing an eddy current damper as shown in fig. 1(a). The concept of eddy current damping is explained by Sodano [11] and Ebrahimi et al. [12].

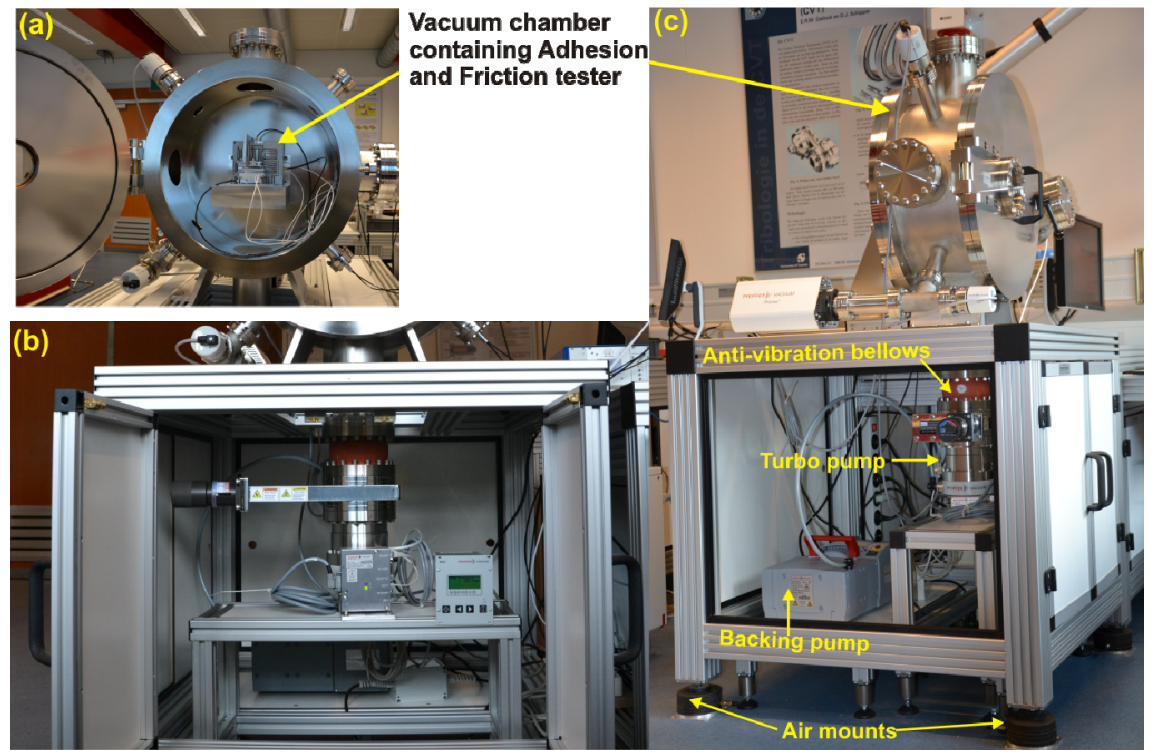

Figure 3: (a) Adhesion and friction tester mounted inside the vacuum chamber. (b) Mounting and placement of vacuum pumps and vacuum chamber. (c) Description of components mounted to reduce vibrations.

\section{Results and discussion}

Experiments were performed with two kinds of contacting bodies. The first type depicts a single asperity contact with a silicon ball and a smooth float glass flat surface. The second type was a rough Zirconia $\left(\mathrm{ZrO}_{2}\right)$ ball and a rough $\mathrm{ZrO}_{2}$ flat surface representing a multi asperity contact. These materials were used to perform adhesion and friction experiments both in ambient and high vacuum conditions. The material properties of the materials used have been reported by Xu et al. [13] Harnett et al. [14] and Krol and Krol [15] and are shown in table 1.

\subsection{Adhesion force measurements}

Adhesion force measurements were performed with Si-float glass and the $\mathrm{ZrO}_{2}$ $\mathrm{ZrO}_{2}$ combinations both in ambient and vacuum. A typical force-displacement 
Table 1: $\quad$ Material properties of the contacting surfaces.

\begin{tabular}{|c|c|c|c|c|}
\hline Properties & $\begin{array}{c}\text { Elastic modulus } \\
E(\mathrm{GPa})\end{array}$ & $\begin{array}{c}\text { Surface energy } \\
\gamma\left(\mathrm{mJ} / \mathrm{m}^{2}\right)\end{array}$ & $\begin{array}{c}\text { Roughness } R_{q} \\
(\mathrm{~nm})\end{array}$ & $\begin{array}{c}\text { Radius } R \\
(\mathrm{~mm})\end{array}$ \\
\hline Si ball & 168 & $44.1 \pm 3.1^{*}$ & 2 & 5 \\
\hline Float Glass flat & 74 & 83.4 & 1 & $\infty$ \\
\hline $\mathrm{ZrO}_{2}$ ball & 200 & 45.6 & 800 & 0.8 \\
\hline $\mathrm{ZrO}_{2}$ flat & 200 & 45.6 & 400 & $\infty$ \\
\hline
\end{tabular}

Surface energy of $\mathrm{SiO}_{2}$ because of oxide layers present on the surface

curve for the Si-float glass combination in ambient is shown in fig. 4. This forcedisplacement curve represents the applied normal load plotted against the vertical displacement of the $\mathrm{Z}$ stage. In fig. 4 the three different areas of the forcedisplacement curve are shown. In fig. 4(a) two distinctive regions of the forcedisplacement curve are shown which represents before and after the contact situations. The $\mathrm{Z}$ stage starts moving downward from the home position, until the desired normal load is applied after making the contact. This section of the curve is called "approach" and is represented by the dotted arrow. The part of the measurement where the contact is made and the flat surface is loaded and unloaded is shown in fig. 4(b). The hysteresis in the loading-unloading loop is typical adhesion hysteresis because the work needed to separate two surfaces is greater than the work that was gained by bringing them together as explained by Yoshizawa et al. [16] and Lui and Bhushan [17].

The exact pull-off point is shown in fig. 4(c) which is zoomed in area of point "P" in fig 4(b). The contact is broken from the surface at the "Pull-off point" when the $Z$ stage is moving upward as shown in fig. 4(c). This section of the curve is called "retract" and is represented by the dashed arrow. The negative value of the force represents the adhesion force present in this particular system and environment. Furthermore, it can be seen from the graphs that the flat surface has been loaded with $11 \mathrm{mN}$ by the silicon ball. The corresponding adhesion force is about $518 \mu \mathrm{N}$. After the breakaway the dynamic effects in the measurement data can be seen.

With the same system, the adhesion force measurements in vacuum have also been performed and similar force-displacement curves at a pressure of $10^{-6} \mathrm{mbar}$ are shown in fig. 5. A significant difference in the pull-off force is observed when measured in vacuum compared to ambient. It can be seen that the pull-off force has significantly reduced in vacuum conditions because different magnitude of meniscus and van der Waals forces are contributing to the adhesion force (Grobelny et al. [2]). The flat surface is loaded with approx. $8.7 \mathrm{mN}$ and the corresponding pull-off force is approx. $17.6 \mu \mathrm{N}$.

Adhesion force measurements both in vacuum and ambient were also performed with a rough $\mathrm{ZrO}_{2}$ ball against a rough $\mathrm{ZrO}_{2}$ flat surface. The pull-off force in both environments was significantly lower than the single asperity contact. The reason for this is because there are small microcontacts present in the contact and therefore, the real area of contact is much smaller than the 

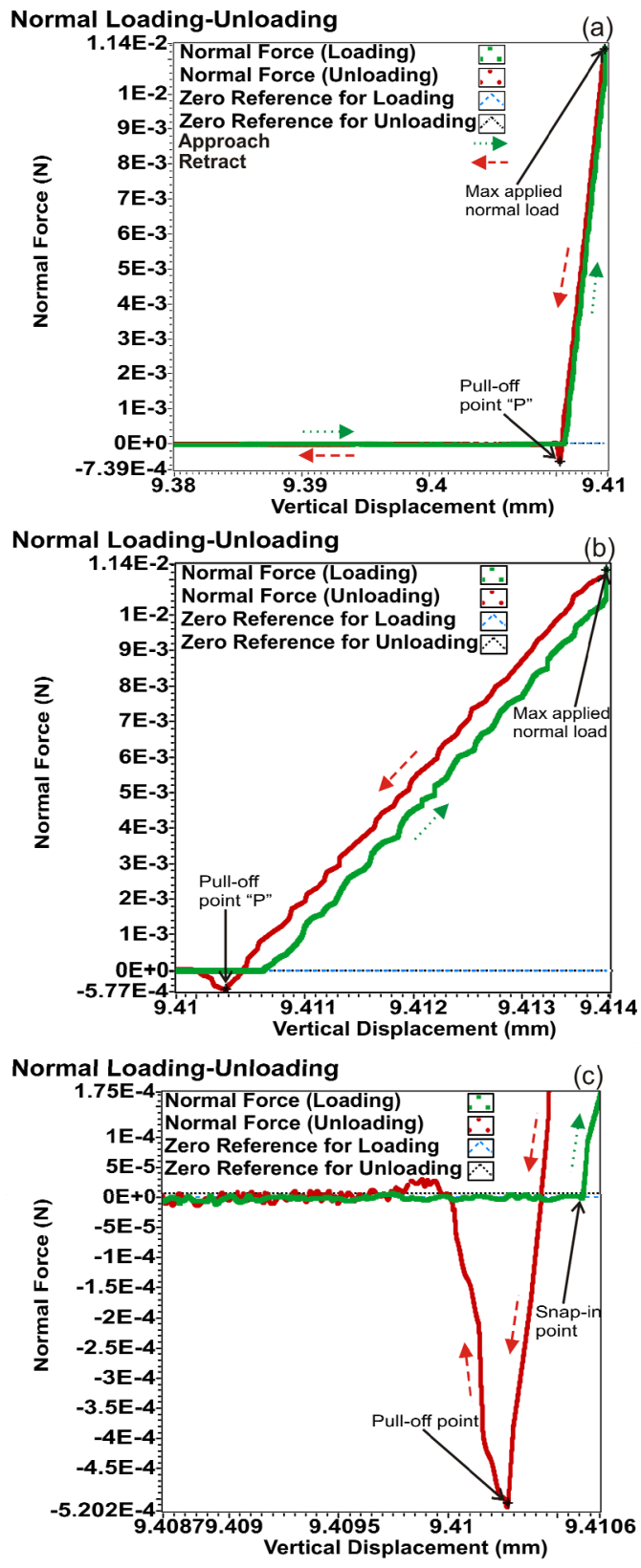

Figure 4: A typical force-displacement curve between vertical displacement and normal load to measure adhesion force. (a) Before and after contact situation. (b) Slope of the loading-unloading curve. (c) Point " $P$ " zoomed in showing pull-off and snap-in points. 


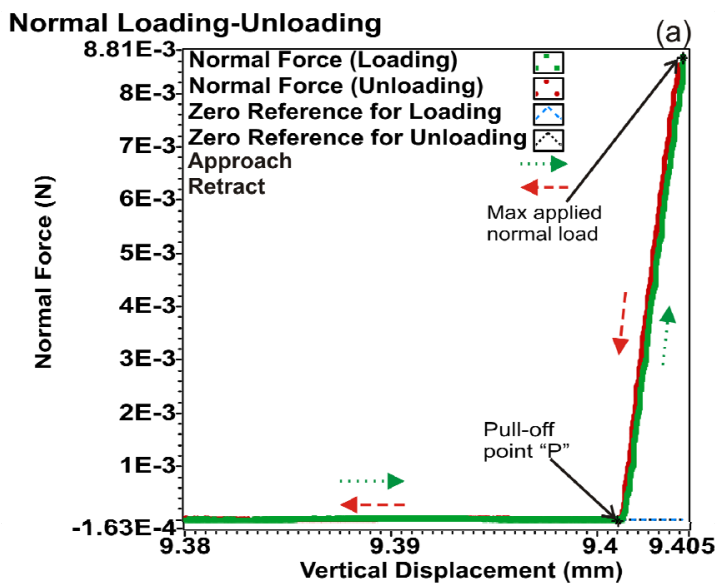

Normal Loading-Unloading
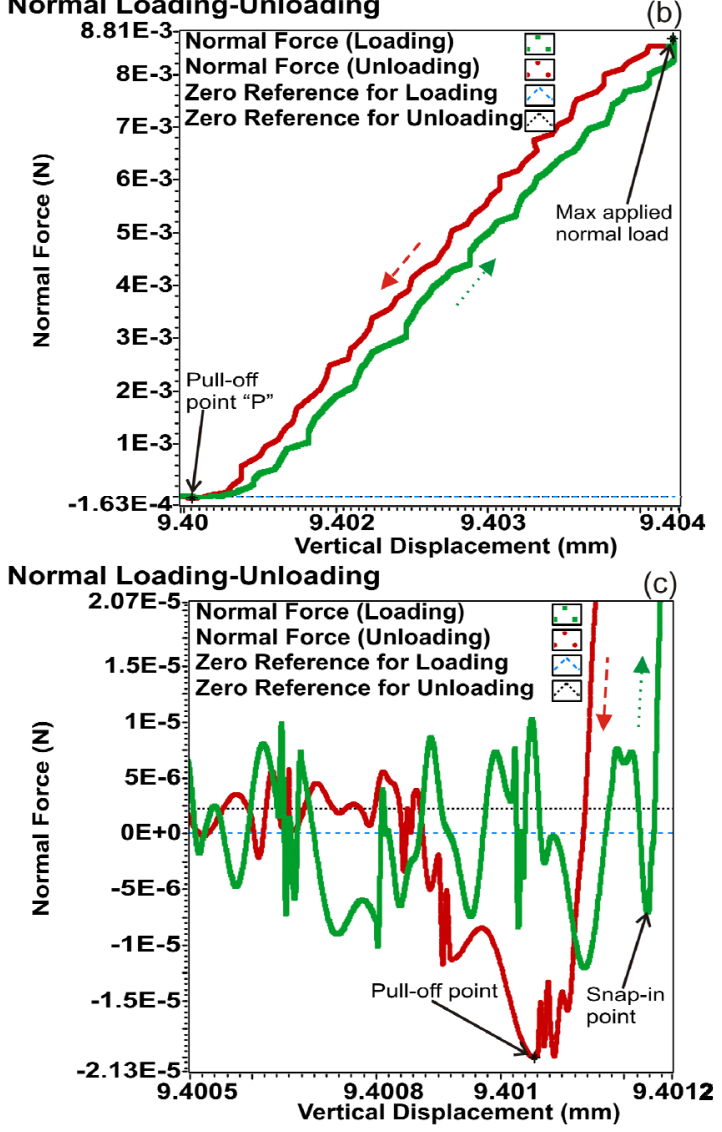

Figure 5: Adhesion force measurement between Si ball and glass flat surface in vacuum. (a) Before and after contact situation. (b) Loadingunloading cycle of the measurement. (c) Pull-off and snap-in point. 
nominal area of contact. The capillary force and the van der Waals force scales linearly with the radius of the spherical surface, see Israelachivili [1]. Similar results have been reported by van Zwol et al. [18].

\subsection{Friction force measurements}

Experiments were performed to study the frictional behaviour of the Si-Glass interface. A typical force-displacement curve for measuring the friction force is shown in fig. 6 . The force measured with the force measuring mechanism in the tangential direction is plotted against the horizontal displacement of the X stage. The X stage moves towards right to load the contact tangentially as shown in fig. 1(a). In fig. 6(a) a friction loop is shown for measurements performed in ambient conditions. The flat surface is loaded with the desired load with the help of $\mathrm{Z}$ stage and then the tangential load is applied with the help of X stage. Similar friction force loops have been measured in high vacuum conditions and an example is shown in fig. 6(b). Moreover, the static and dynamic friction regimes are distinctively seen along with the preliminary displacement $\delta_{\mathrm{a}}$ and gross slip regions. In ambient conditions in this particular case the applied normal load is $6.5 \mathrm{mN}$ which resulted in $3.3 \mathrm{mN}$ of friction force and $2 \mu \mathrm{m}$ of preliminary displacement. However, in the vacuum environment the applied normal load is $9 \mathrm{mN}$ and a friction force of $2 \mathrm{mN}$ with a preliminary displacement of $0.7 \mu \mathrm{m}$.

The coefficient of friction (COF) and preliminary displacement $\left(\delta_{a}\right)$ as a function of applied normal load both in ambient and high vacuum conditions are shown in fig. 7. A significant decrease is seen when the same system is operated in the high vacuum environment both for the preliminary displacement and the COF. According to Hertz theory the contact area is proportional to the applied normal load to the power $2 / 3$ as shown in eqn. (2) and the friction force is given as shown in eqn. (3).

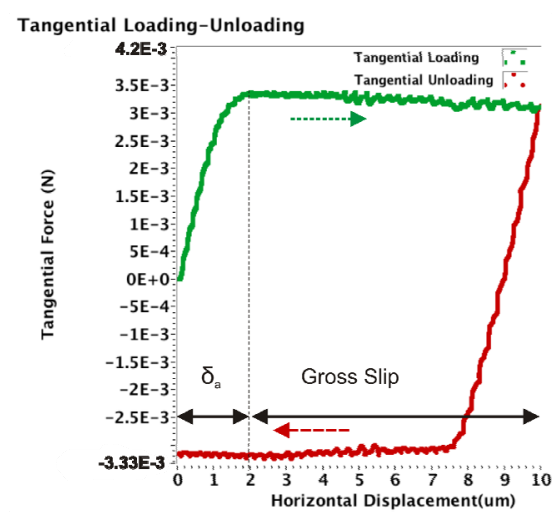

(a)

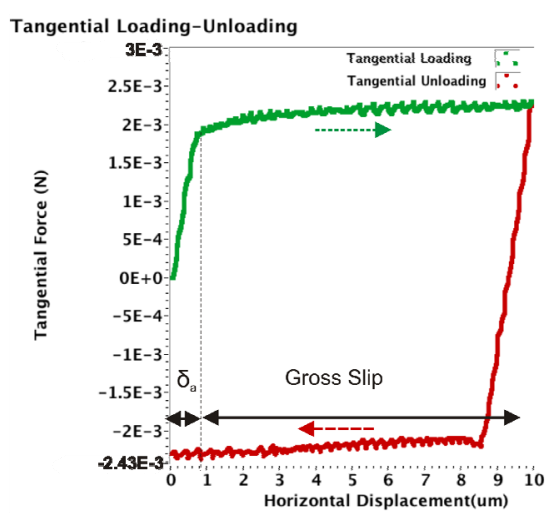

(b)

Figure 6: Typical friction force loops measuring friction force and preliminary displacement for Silicon-Glass interface in (a) ambient and (b) vacuum. 
(a)

(a) Coefficient of Friction vs Normal Load

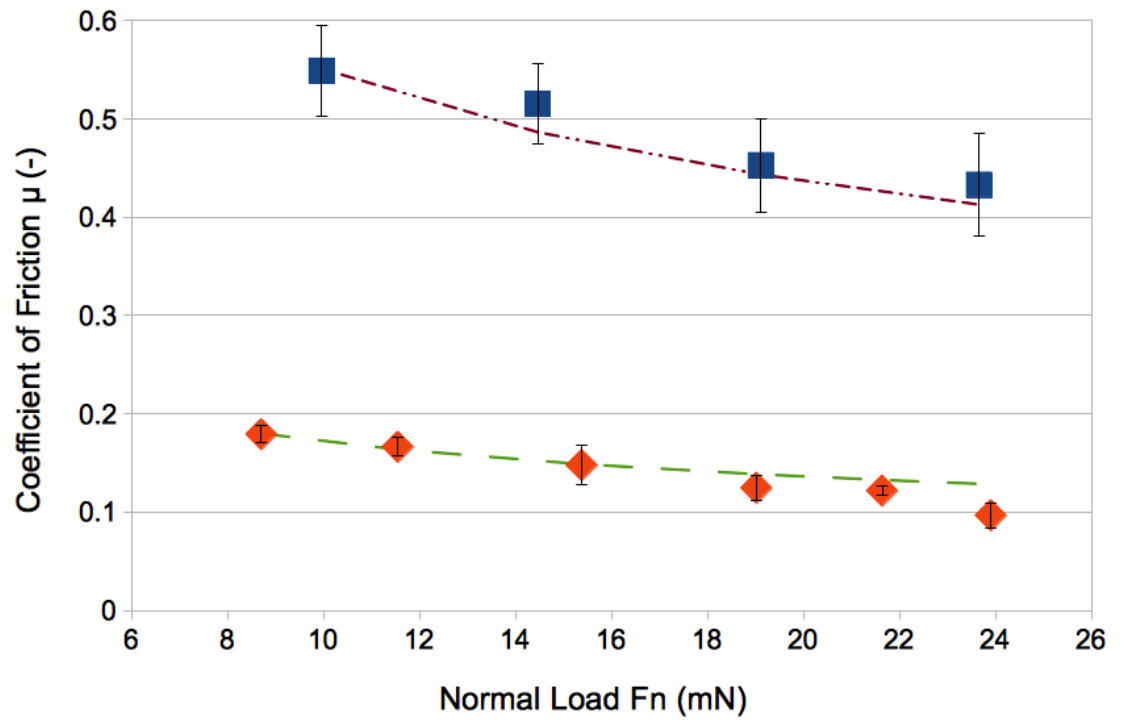

(b)

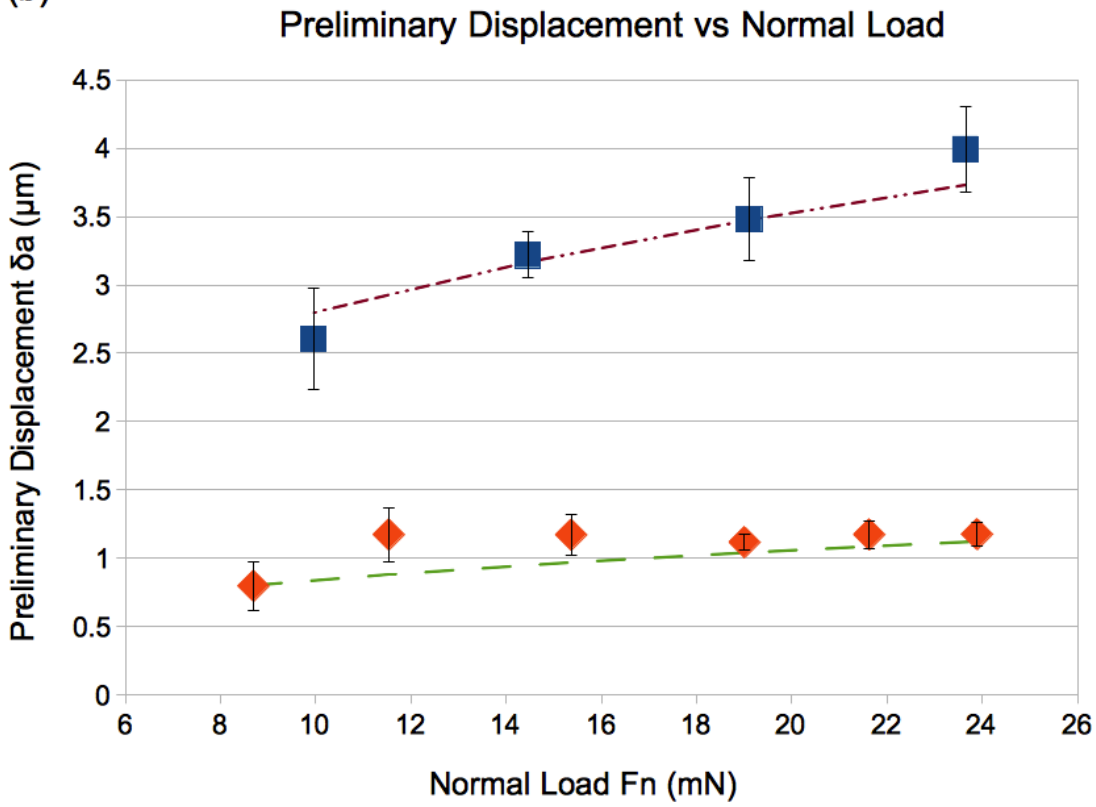

Figure 7: (a) Coefficient of friction and (b) Preliminary displacement against normal load measured both in ambient $\boldsymbol{\square}$ and high vacuum $\rightarrow$ for Si-Glass system. Power fitting shows the validation of the results with theory. 


$$
\begin{gathered}
A=\pi \cdot\left(\frac{3}{4} \cdot \frac{R}{E^{*}}\right)^{2 / 3} \cdot F_{n}^{2 / 3} \\
F_{t}=\tau \cdot A
\end{gathered}
$$

The coefficient of friction is defined by eqn. (4):

$$
\mu=\frac{F_{t}}{F_{n}}, \Rightarrow \mu \propto \frac{F_{n}^{2 / 3}}{F_{n}}, \mu \propto F_{n}^{-1 / 3} .
$$

Assuming a constant shear stress $\tau$ of the interface the coefficient of friction is proportional to the applied normal load to the power $-1 / 3$ as shown in eqn. (4). The curve fittings in fig. 7(a) show that the measurements are in good agreement with the theory. Similarly, in fig. 7(b) the preliminary displacement $\delta_{\mathrm{a}}$ is plotted against the applied normal load. According to Mindlin [19] the microslip occurs before the gross slip when the tangential load is applied on the contacting bodies. This microslip here is characterised as preliminary displacement and is given by Galligan and McCullough [20]:

$$
\delta_{a}=\frac{F_{t}}{8 \cdot a} \cdot\left(\frac{2-v_{1}}{G}\right), \Rightarrow \delta_{a} \propto \frac{\mu \cdot F_{n}}{a}, \delta_{a} \propto \frac{F_{n}^{-1 / 3} \cdot F_{n}}{F_{n}^{1 / 3}}, \delta_{a} \propto F_{n}^{1 / 3} .
$$

where, $\delta_{\mathrm{a}}$ is directly proportional to the tangential load $F_{t}$ and inversely proportional to the contact radius $a$. The COF is proportional to the applied normal load to the power $-1 / 3$ and the contact radius is proportional to the applied normal load to the power $1 / 3$, this gives us the $\delta_{\text {a }}$ proportional to the applied normal load to the power $1 / 3$ as shown in eqn. (5). The experimental results shown in fig. 7(b) are also in agreement with the theory since the increasing trend is following the $1 / 3$ power of normal load.

\section{Conclusions}

The design and construction of the Vacuum Adhesion Friction Tester has been discussed. The test rig is capable of measuring micro adhesion and friction with an accuracy of $5 \mu \mathrm{N}$ in ambient as well as in high vacuum conditions. The setup is able to measure adhesion and friction force for both single asperity and multi asperity contacts. The experimental results for the single asperity contact have also been discussed. Force-displacement curves for both adhesion and friction measurements both in ambient and high vacuum conditions have been presented. Adhesion and friction forces are reduced when the same system is operated in high vacuum conditions. The experimental results show good agreement with the basic theories of contact mechanics.

\section{Acknowledgements}

This research was carried out under project number MC7.06284 in the framework of the Research Program of the Materials innovation institute M2 $\mathrm{i}$ 
(www.m2i.nl). Financial support for carrying out this research from the M2i is gratefully acknowledged.

\section{References}

[1] Israelachivili, J. N., Intermolecular and Surface Forces. Academic Press LTD: London and San Diego, Second ed., 1991.

[2] Grobelny, J., Paradeep, N., Kim, D.-I. and Ying, Z.C., Quantification of the meniscus effect in adhesion force measurements, Applied Physics Letters, 88(091906), pp. 1-3, 2006.

[3] Amstrong-Hélouvry, B., Control of machines with friction, Kluwer Academic Publishers: Massachusetts and Dordrecht, 1991.

[4] Capone, G., Della Valle, S., Guida, D. and D'Agostino, V., On the frictionposition control relationship, Proceedings of the Institution of Mechanical Part J: Engineers. Journal of Engineering Tribology, 211(2), pp. 129-137, 1997.

[5] Feldman, K., Fritz, M., Hahner, G., Marti, A. and Spencer, N.D., Surface Forces, Surface Chemistry and Tribology. Tribology International, 31 (13), pp. 99-105, 1998.

[6] Xiao, X. and Qian, L., Investigation of humidity-dependent capillary force, Langmuir, 16, pp. 8153-8158, 2000.

[7] Bhushan, B., Adhesion and stiction: Mechanisms, measurement techniques, and methods for reduction, Journal of Vacuum Science and Technology B, 21(6), pp. 2262-2292, 2003.

[8] Johnson, K.L. and Greenwood, J.A., An adhesion map for the contact of elastic spheres, Journal of Colloid and Interface Science, 192, pp. 326-333, 1997.

[9] Awtar, S., Synthesis and analysis of parallel kinematic XY flexure mechanism, Doctoral Dissertation, Cambridge Massachusetts, pp. 23-42, 2004.

[10] Agilent Technologies Vacuum Technologies - Product Catalogue 2010, Catalogue for Turbo Pumps, www.chem.agilent.com

[11] Sodano, H.A., Development of novel eddy current dampers for the suppression of structural vibrations, Doctoral Dissertation, Blacksburg Virginia, pp. 44-94, 2005.

[12] Ebrahimi, B., Khamesee, M.B. and Golnaraghi, F., A novel eddy current damper: theory and experiment, Journal of Physics D: Applied Physics, 42(075001), pp. 1-6, 2009.

[13] Xu, D., Liechti, K. M. and Ravi-Chandar, K., On the modified tabor parameter for the JKR-DMT transition in the presence of a liquid meniscus, Journal of Colloid and Interface Science, 315, pp. 772-785, 2007.

[14] Harnett E.M., Alderman J. and Wood T., The surface energy of various biomaterials coated with adhesion molecules used in cell culture, Colloids and Interface Science B: Biointerfaces, 55, pp. 90-97, 2007. 
[15] Krol, P. and Krol, B., Determination of free surface energy values for ceramic materials and polyurethane surface-modifying aqueous emulsions. Journal of the European Ceramic Society, 26, pp. 2241-2248, 2006.

[16] Yoshizawa, H., Chen, Y.L. and Israelachvili, J., Fundamental mechanisms of interfacial friction 1. Relation between adhesion and friction, Journal of Physical Chemistry, 97(16), pp. 4128-4140, 1993.

[17] Liu, L. and Bhushan B., Adhesion and friction studies of microelectromechanical systems/ nanoelectromechanical systems materials using a novel microtriboapparatus, Journal of Vacuum Science and Technology A, 21(4), pp. 1528-1538, 2003.

[18] van Zwol, P.J., Palasantzas, G., and De Hosson, J.Th.M., Influence of roughness on capillary forces between hydrophilic surfaces, Physical Review E, 78(031606), pp. 1-6, 2008.

[19] Mindlin, R.D., Compliance of elastic bodies in contact, ASME Journal of Applied Mechanics, 16, pp. 259-268, 1949.

[20] Galligan, J.M. and McCullough, P., On the nature of static friction, Wear, 105, pp.337-340, 1985. 